\title{
A 0.6-V 1.6-GHz 8-phase all digital PLL using multi-phase based TDC
}

\author{
Yo-Hao Tu${ }^{1}$, Jen-Chieh Liu ${ }^{2 a)}$, Kuo-Hsing Cheng ${ }^{1}$, \\ Hong-Yi Huang ${ }^{3}$, and Chang-Chien $\mathrm{Hu}^{1}$ \\ ${ }^{1}$ Department of Electrical Engineering, National Central University, \\ No. 300, Zhongda Rd., Jhongli District, Taoyuan City 32001, Taiwan \\ 2 Department of Electrical Engineering, National United University, \\ No. 2, Lienda, Miaoli City 36003, Taiwan \\ ${ }^{3}$ Graduate Institute of Electrical Engineering, National Taipei University, \\ No. 151, University Rd., San Shia District, New Taipei City 23741, Taiwan \\ a)jcliu@nuu.edu.tw
}

\begin{abstract}
This paper proposes an 8-phase all-digital phase-locked loop (ADPLL) for a low supply voltage application. The proposed multi-phase digitally controlled oscillator (MP-DCO) employs two sub-feedback loops at high operational frequencies. The proposed multi-phase-based time-to-digital converter (MP-TDC) uses the multi-phase scheme, which reduces its area, and uses a time amplifier to extend the timing resolution. With a low supply voltage, the DCO and the sense-amplifier based delay flip-flop (SA-DFF) use bulk-controlled techniques to improve the performance at high operational frequencies and setup/hold times, respectively. When the ADPLL output is $1.6 \mathrm{GHz}$ at $0.6 \mathrm{~V}$, the RMS and peak-to-peak jitters are $3.8 \mathrm{ps}$ and $33.7 \mathrm{ps}$, respectively. The power consumption and core area are $9.1 \mathrm{~mW}$ at $1.6 \mathrm{GHz}$ and $0.036 \mathrm{~mm}^{2}$ in a $90 \mathrm{~nm}$ CMOS process, respectively. Thus, this clock generator is useful for low power systems.
\end{abstract}

Keywords: all-digital phase-locked loop (ADPLL), digital controlled oscillator (DCO), time-to-digital converter (TDC), time amplifier (TA), low supply voltage, multi-phase outputs

Classification: Integrated circuits

\section{References}

[1] C.-T. Lu, H.-H. Hsieh and L.-H. Lu: IEEE Trans. Circuits Syst. I, Reg. Papers 57 (2010) 793. DOI:10.1109/TCSI.2009.2028596

[2] M. Lee, M. E. Heidari and A. A. Abidi: IEEE J. Solid-State Circuits 44 (2009) 2808. DOI: $10.1109 /$ JSSC.2009.2028753

[3] S.-K. Lee, Y.-H. Seo, H.-J. Park and J.-Y. Sim: IEEE J. Solid-State Circuits 45 (2010) 2874. DOI:10.1109/JSSC.2010.2077110

[4] J. A. Tierno, A. V. Rylyakov and D. J. Friedman: IEEE J. Solid-State Circuits 43 (2008) 42. DOI:10.1109/JSSC.2007.910966

[5] J. Lin, B. Haroun, T. Foo, J.-S. Wang, B. Helmick, S. Randall, T. Mayhugh, C. Barr and J. Kirkpatric: ISSCC Dig. Tech. Papers (2004) 488. DOI:10.1109/ ISSCC.2004.1332807 
[6] W. Liu, W. Li, P. Ren, C. Liu, S. Zhang and Y. Wang: IEEE J. Solid-State Circuits 45 (2010) 314. DOI:10.1109/JSSC.2009.2038127

[7] S.-Y. Yang, W.-Z. Chen and T.-Y. Lu: IEEE J. Solid-State Circuits 45 (2010) 578. DOI:10.1109/JSSC.2009.2039530

[8] E. Temporiti, C. Weltin-Wu, D. Baldi, M. Cusmai and F. Svelto: IEEE J. SolidState Circuits 45 (2010) 2723. DOI:10.1109/JSSC.2010.2077370

[9] M. S.-W. Chen, D. Su and S. Mehta: IEEE J. Solid-State Circuits 45 (2010) 2819. DOI:10.1109/JSSC.2010.2074950

[10] J. Yang, J.-Y. Lee, S.-J. Lim and H.-M. Bae: IEEE Trans. Circuits Syst. II, Exp. Briefs 61 (2014) 880. DOI:10.1109/TCSII.2014.2356893

[11] H.-Y. Huang and F.-C. Tasi: DDECS (2009) 28. DOI:10.1109/DDECS.2009. 5012093

[12] V. Kratyuk, P. K. Hanumolu, U.-K. Moon and K. Mayaram: IEEE Trans. Circuits Syst. II, Exp. Briefs 54 (2007) 247. DOI:10.1109/TCSII.2006.889443

[13] S. Narendra, J. Tschanz, J. Hofsheier, B. Bloechel, S. Vangal, Y. Hoskote, S. Tang, D. Somasekhar, A. Keshavarzi, V. Erraguntla, G. Dermer, N. Borkar, S. Borkar and V. De: ISSCC Dig. Tech. Papers (2004) 156. DOI:10.1109/ISSCC. 2004.1332641

[14] K.-H. Cheng, C.-C. Hu, J.-C. Liu and H.-Y. Huang: DDECS (2010) 285. DOI:10.1109/DDECS.2010.5491766

[15] K.-H. Cheng, J.-C. Liu and H.-Y. Huang: IEEE Trans. Circuits Syst. II, Exp. Briefs 59 (2012) 888. DOI:10.1109/TCSII.2012.2231021

\section{Introduction}

For wireless transceivers, the most common way to recover a received data signal is a phase-locked loop (PLL)-based clock and data recovery (CDR). The digital phase-locked loop (DPLL) consumes less power than the analog one. The digital circuit has a small area, a short-locking time, inherent noise immunity and scalability with process shrink. Energy saving is an impact factor for system-onchip systems. For mobile wireless communications, the battery lifetime is limited by the power consumption of the electronics circuits. In order to minimize the required dc power, operating the circuits at a reduced supply voltage is apparently an effective approach [1]. Furthermore, it can be suitable for solar cell systems and portable devices. Generally, the bottlenecks of a digital PLL include: One is the timing resolution of the time-to-digital converter (TDC). The timing resolution, linearity and measured range of the TDC can be improved using time amplifier (TA) and coarse-fine tuning techniques. In previous studies [2] and [3], a time amplifier has TDC quantization issues. The other is operational frequency and multi-phase outputs of the digital controlled oscillator (DCO). In [4], the inverterbased ring oscillator technique applied the wide ranges of operational frequencies and supply voltages. DPLL can be insensitive to process, voltage, and temperature (PVT) variations in [5] and [6]. LC-tank DCOs achieve a higher operational frequency and lower phase noise as shown in [7] and [8].

Multi-phase clocks require its components operate at a frequency that equals the bit rate. A 10+GBs clock and CDR incorporate a quarter-rate phase detector or a multi-phase clock source to re-time and de-multiplex a 10+GBs input data signal. Therefore, a multi-phase clock is useful for a receiver. Three useful methods for 
multi-phase outputs have been disused in $[9,10,11]$. First, the conventional scheme uses an inverter chain, which is easy to implement, but its operational frequency depends on the number of stages in the inverter chain [9]. The second method uses a phase interpolator [10], whose circuit improves the phase resolution. The third approach uses an oscillator with a k-stage sub-feedback loop to increase operational frequency and generate multi-phase outputs [11]. It also operates at a low supply voltage as a gigahertz oscillator with multi-phase outputs.

In this work, an all-digital PLL (ADPLL) is operated at a high frequency with multi-phase outputs under a low supply voltage. Its architecture offers three benefits. First, the multi-phase DCO (MP-DCO) with two sub-feedback loops yield a high operational frequency at a low supply voltage. Second, the multi-phasebased TDC (MP-TDC) reuses the MP-DCO outputs for sampled clock, reducing the area of TDC. Finally, a DCO and a TDC use bulk-controlled techniques to increase the operational frequency and timing resolution, respectively. These three benefits are exploited to implement a $0.6 \mathrm{~V} 1.6 \mathrm{GHz}$ ADPLL with 8-phase outputs.

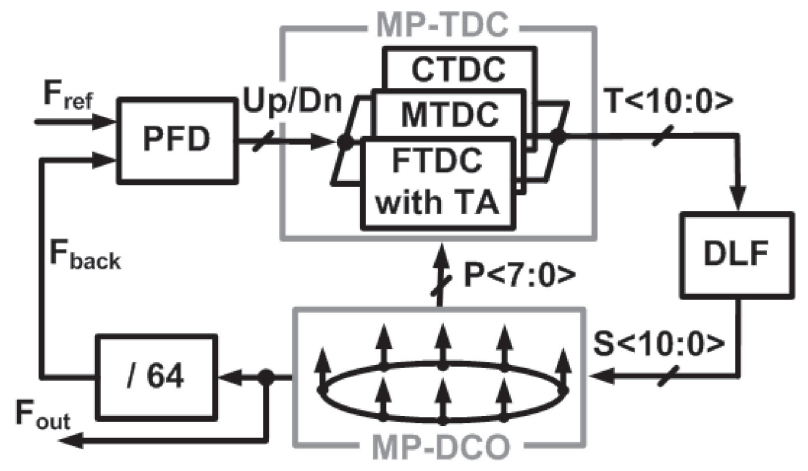

Fig. 1. Block diagram of the proposed 8-phase ADPLL.

\section{Architecture}

\section{A. Architecture of 8-phase ADPLL based on MP-TDC}

Fig. 1 displays a block diagram of the ADPLL. The operational frequency and phase acquisition aligns the DCO clock edge $\left(F_{\text {out }}\right)$ to the reference clock edge $\left(F_{\text {ref }}\right)$. Thus, the feedback clock edge $\left(F_{\text {back }}\right)$ is locked to the reference clock through the control of the divider value $(N)$. The phase frequency detector (PFD) creates the timing difference ( $U p$ and $D n)$ to provide the 11-bit MP-TDC. The phase and frequency errors are detected and digitized by the MP-TDC. The MPTDC digital code is applied to the digital loop filter (DLF) to align the DCO frequency. Accordingly, the DCO frequency follows the algorithm of the DLF.

The closed-loop stability of the ADPLL is characterized using a linear s-domain model [12]. A type-II second-order analog PLL is used to analyse the frequency response of ADPLL. The algorithm of the digital loop filter uses a proportionalintegral scheme. The loop gain of the ADPLL is calculated as:

$$
G(s)=\frac{1}{N} \frac{T_{R E F}}{\Delta_{\mathrm{MP}-\mathrm{TDC}}} \frac{K_{M P-D C O}}{s} \frac{s+\omega_{z}}{s} R
$$


equal to $1 /(R C) . K_{M P-D C O}$ and $N$ represent the DCO gain and the divisor of the divider, respectively. The DLF consists of a proportional path $(\alpha)$ and an integral path $(\beta)$. In terms of the parameters of the analog loop filter $R$ and $C, D L F(s)$ can be written as $R+1 / s C$ which is applied to design parameters $\alpha$ and $\beta$. Thus, $\alpha$ equals $R-(T s / 2 C)$ and $\beta$ equals $T s / 2 C$. Ts is the sampled ratio of DLF.

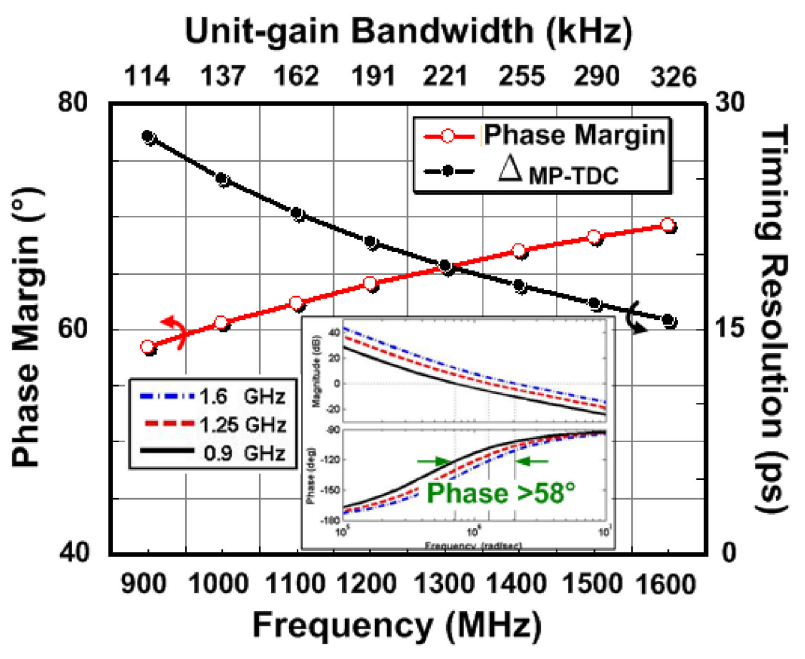

Fig. 2. Simulated behavior in Matlab.

Table I. Parameters of ADPLL at $0.6 \mathrm{~V}$

\begin{tabular}{|c|c|c|c|c|c|}
\hline $\begin{array}{c}11 \text {-bit DLF } \\
\alpha=2^{-4}, \beta=2^{-8}\end{array}$ & $\begin{array}{c}\omega_{\mathrm{UGBW}} \\
(\mathrm{kHz})\end{array}$ & $\begin{array}{c}\text { PM } \\
\left({ }^{\circ}\right)\end{array}$ & $\begin{array}{c}\mathrm{K}_{\mathrm{MP}-\mathrm{DCO}} \\
(\mathrm{MHz} / \mathrm{LSB})\end{array}$ & $\begin{array}{c}\Delta_{\mathrm{MP}-\mathrm{TDC}} \\
(\mathrm{ps})\end{array}$ & $\mathrm{N}$ \\
\cline { 1 - 5 } $\mathrm{F}_{\text {out }}=900 \mathrm{MHz}$ & 114 & 58.4 & 0.24 & 27.8 & \multirow{2}{*}{64} \\
\cline { 1 - 5 } $\mathrm{F}_{\text {out }}=1.6 \mathrm{GHz}$ & 326 & 69.2 & 0.77 & 15.6 & \\
\hline
\end{tabular}

In this work, the phase margin $(P M)$ and unity gain-bandwidth $\left(\omega_{U G B W}\right)$ of ADPLL are shown in Fig. 2. Table I lists the ADPLL parameters. The timing resolution of MP-TDC follows the DCO output frequency. Hence, a higher DCO output frequency yields a higher timing resolution of MP-TDC. The sampled clock has twice the period of the reference clock is the DLF parameter. The relationship between the timing resolution of MP-TDC and the DCO output period is fixed. As the MP-DCO output frequency increases, the phase margin of ADPLL increases according to Eq. (1). In Fig. 2, the phase margin of ADPLL is larger than $58^{\circ}$ from $900 \mathrm{MHz}$ to $1.6 \mathrm{GHz}$, as determined in Matlab.

\section{B. Proposed MP-DCO with 3-stage sub-feedback scheme}

The sub-feedback loop technique for PLL as analog type was proposed in [11]. It usefully increases operational frequency with multi-phase outputs. They also analyzed an n-stage ring oscillator with 3-stage sub-feedback loops [11]. The block diagram of MP-DCO is illustrated in Fig. 3. The 8-stage ring oscillator with two sub-feedback loops is adopted. The main loop circuit is designed for 8-phase outputs. The first loop circuit adopts the digital controlled ring-based delay line (RB-DL) and MOS varactors to tune the range of DCO frequencies. The second loop circuit uses three inverters to increase the operational frequency. 


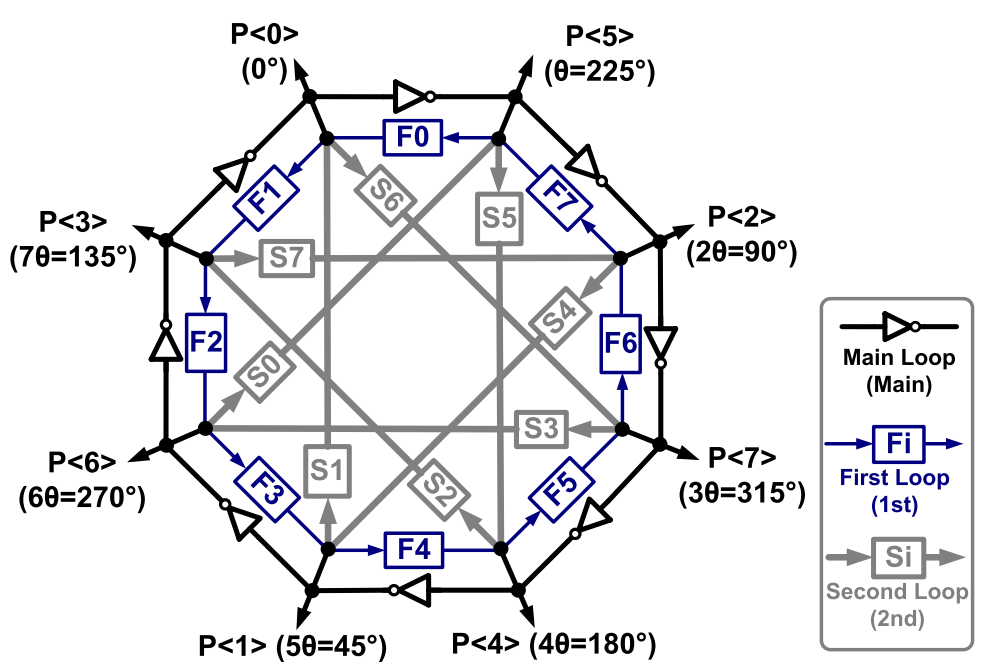

Fig. 3. Block diagram of proposed MP-DCO.

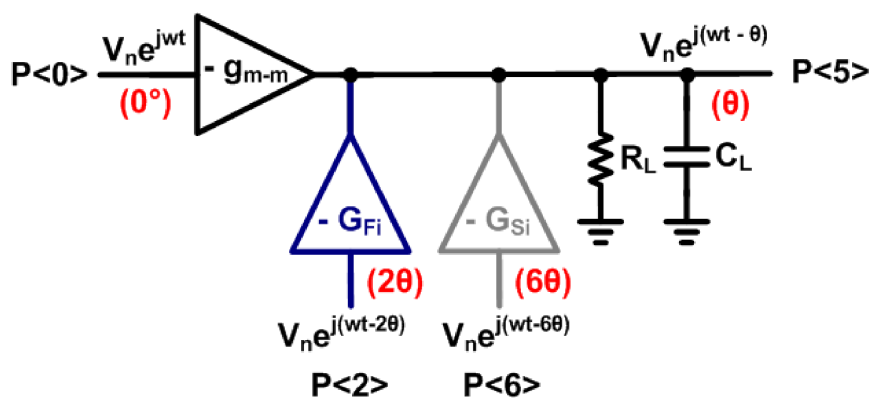

Fig. 4. Linear model of proposed MP-DCO.

Fig. 4 shows the DCO model between $P\langle 0\rangle$ and $P\langle 5\rangle$. Based on assumption that the MP-DCO outputs are sinusoidal waveforms in a steady state, all MP-DCO outputs have the same amplitude. The initial node is $V_{n} e^{j \omega t}$ and the next node is $V_{n} e^{j(\omega t-\theta)}$. The equation of the next node $(P\langle 5\rangle)$ can be expressed as:

$$
\begin{aligned}
& V_{n} e^{j(\omega t-\theta)}= \\
& \quad\left(-g_{m-m} V_{n} e^{j \omega t}-G_{F i} V_{n} e^{j(\omega t-2 \theta)}-G_{S i} V_{n} e^{j(\omega t-6 \theta)}\right)\left(R_{L} / / \frac{1}{j \omega C_{L}}\right)
\end{aligned}
$$

where $g_{m-m}$ is the transconductance of an inverter in the main loop circuit. $G_{F i}$ and $G_{S i}$ are the gain of the first loop circuit and the second loop circuit, respectively. Also, $R_{L}$ and $C_{L}$ are the loading capacitance and equivalent impedance at the output node, respectively. Thus, the approximate oscillation frequency can be calculated as:

$$
\omega=\frac{1}{R_{\mathrm{L}} C_{\mathrm{L}}}\left(\frac{g_{m-m} \operatorname{Sin} \theta-G_{F i} \operatorname{Sin} \theta-G_{S i} \operatorname{Sin} 5 \theta}{g_{m-m} \operatorname{Cos} \theta+G_{F i} \operatorname{Cos} \theta+G_{S i} \operatorname{Cos} 5 \theta}\right)
$$

where $\theta$ is $225^{\circ}$. The sizes of the main loop circuit, the first loop circuit and the second loop circuit are approximate in the ratio $2: 1: 1$. Therefore, the operational frequency of the MP-DCO can be calculated. Fig. 5 shows the theoretical analysis and simulated results for the range of MP-DCO frequencies. The operational frequency of the DCO can be aligned using the first loop circuit and the MOS varactors at the output loading. Thus, the gain of the first loop circuit $\left(G_{F i}\right)$ is used in the coarse-tuning stage and the MOS varactor $\left(C_{L}\right)$ is used in the fine-tuning 
stage. The coarse-tuning stage and fine-tuning stage are 2-bit and 9-bit, respectively. $G_{F i}$ assumes a timing resolution of $0.2 G_{F i}$ for each code and $\mathrm{C}$ has a tuning range of $0.32 \mathrm{C}_{L}$, as shown in Fig. 5. The theoretical analysis and the circuit simulation show the same trend. At higher operational frequencies, the theoretical and simulated results match perfectly since the output signal is a sinusoidal waveform. At lower operational frequencies, the outputs are more like square waveforms. The output loading capacitance at a lower operational frequency is larger, because the fine-tuning stage increases the output loading to achieve a lower operational frequency. The linearity of the operational frequency worsens at higher operational frequencies. The relationship between the phase position and the output phase of the MP-DCO was shown in Fig. 3. The relationship that describes the phase position is:

$$
P\langle i\rangle=P\left\langle\frac{\theta_{P\langle i\rangle}}{45^{\circ}}\right\rangle
$$

Therefore, the phase difference between $P\langle i\rangle$ and $P\langle i+1\rangle$ is $45^{\circ}$.

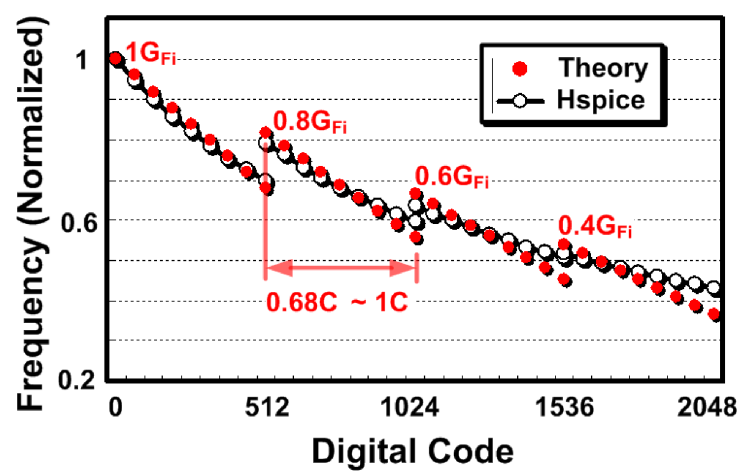

Fig. 5. Operational frequency curve of MP-DCO obtained by theory analysis from simulated results.

\section{Circuit implementation}

\section{A. First and second loop circuits of 8-phase DCO}

The first loop and second loop circuits are shown in Fig. 6(a). The first loop circuit adopts the MOS varactor to increase the timing resolution of the DCO and the ringbased delay line increases the highest operational frequency as shown in Fig. 6(b). The DCO is composed of inverters and NAND gates that are implemented using low threshold (LVT) devices. Fig. 7 shows the comparisons of DCO frequency versus the different conditions of the DCO types. In case A, the conventional DCO adopts only an inverter delay chain for main loop. Thus, the highest operational frequency depends on the inverter delay time. In case B, the forward-biased (FB) scheme increases the highest operational frequency by $52 \%$ [13]. Fig. 8 shows the simulated frequency and power of the DCO under varying of FB voltage. In case C, the DCO adopts the first loop circuit to increase the operational frequency by $234 \%$. Hence, case $\mathrm{C}$ is popular for use in multi-phase and high speed oscillators [11]. In cases D and $\mathrm{E}$, the second loop and RB-DL can extend the highest operational frequency by $9 \%$ and $2 \%$, respectively. Therefore, the 8 -phase DCO with a sub-feedback loop scheme is useful for application that a low supply voltage. 


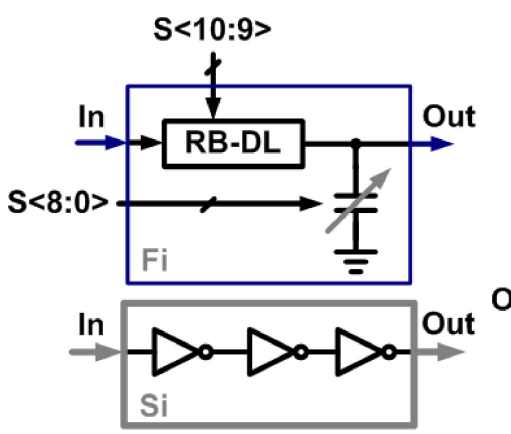

(a)

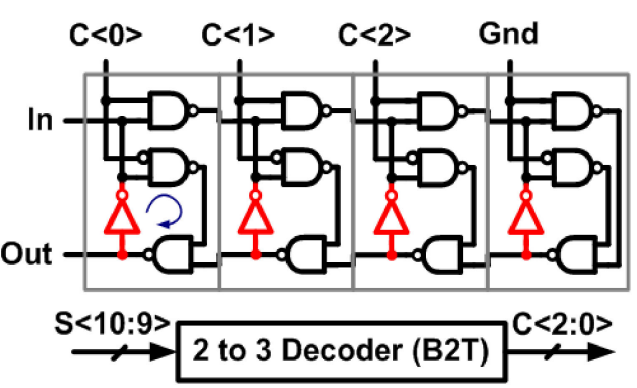

(b)

Fig. 6. (a) Circuits of first loop and second loop (b) Circuitry of RBDL.

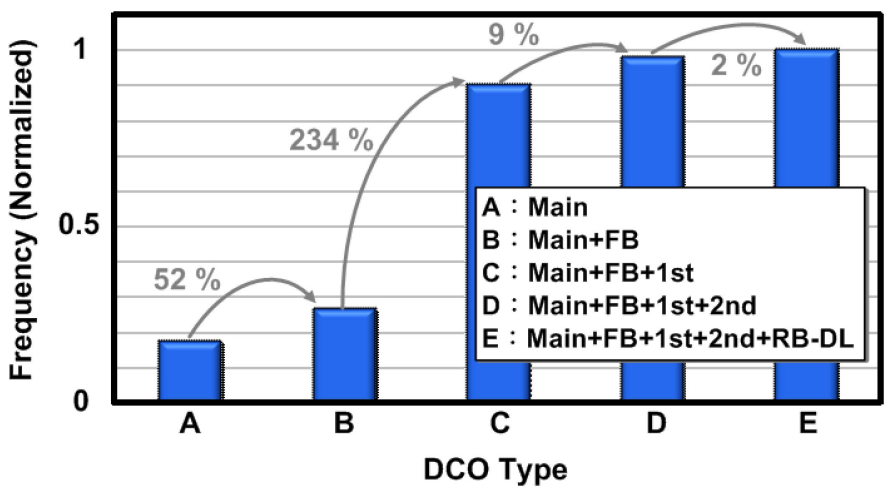

Fig. 7. Highest operational frequency of DCO type.

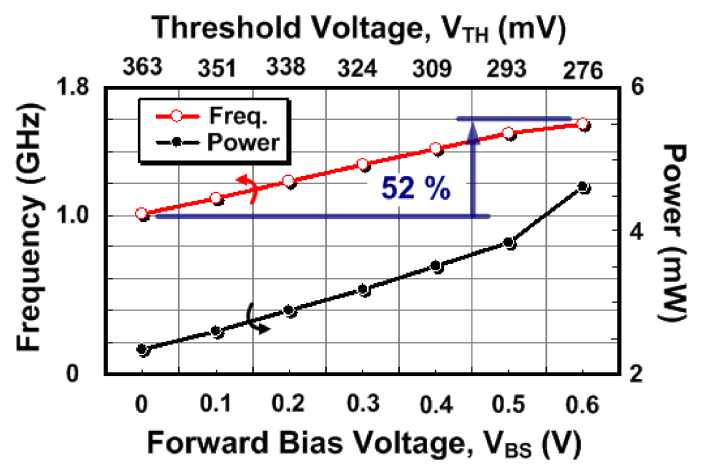

Fig. 8. Parameters of MP-DCO using a forward-biased scheme.

The phase error (PE) characteristic is used to measure the quality of the MPDCO phase resolution. The PE of the MP-DCO can be written as:

$$
P E_{M P-D C O}=\left(\frac{T_{P\langle i+1\rangle-P\langle i\rangle}}{T_{M P-D C O}}\right) \times 360^{\circ}
$$

where $T_{P\langle i+1\rangle-P\langle i\rangle}$ defines the phase error between the $i$-phase position and the $(i+1)$-phase position and $T_{M P-D C O}$ is the period of the MP-DCO in (5). The layout style of the MP-DCO is presented in Fig. 9. To match the 8-phase outputs of MP-DCO, metal wires are designed to have the same loading effect. Fig. 10 shows the post-layout simulation of $P E_{M P-D O C}$ based on a Monte Carlo analysis. The bonding wire effect of $3 \mathrm{nH}, P E_{M P-D O C}$ is smaller than $45 \pm 1.8^{\circ}$ over 100 Monte Carlo samples. 


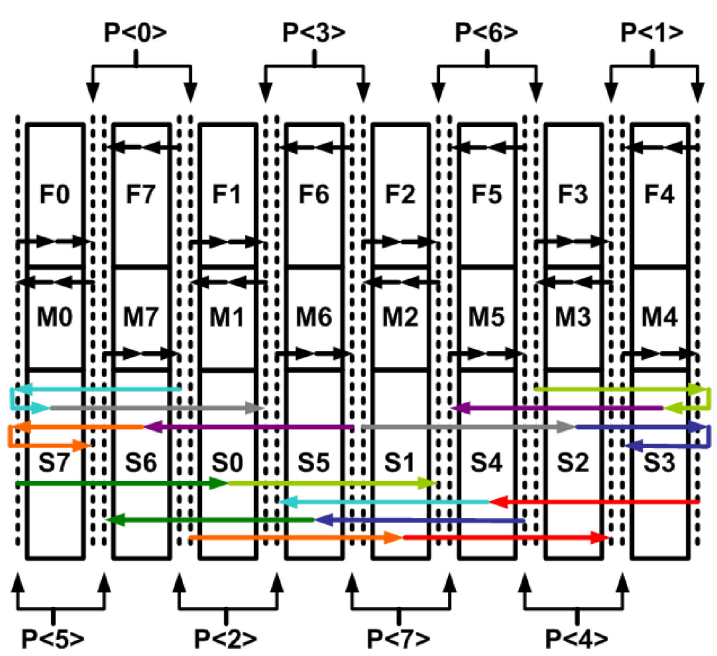

Fig. 9. Layout style of MP-DCO.

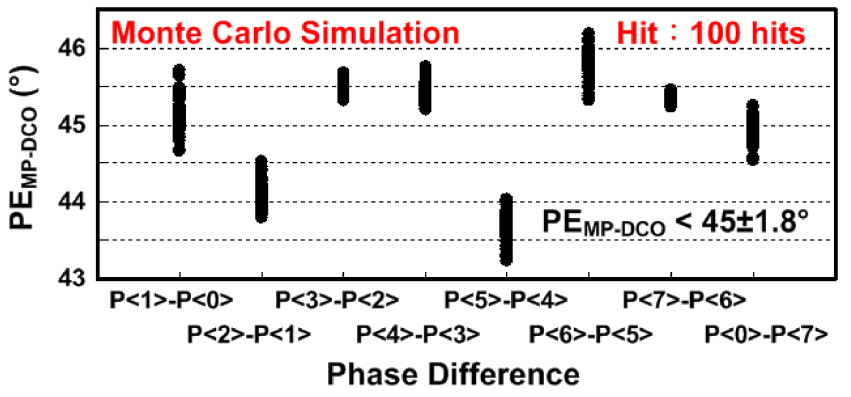

Fig. 10. Simulated phase error of MP-DCO.

\section{B. Multi-phase-based TDC with a TA}

The block diagram of the MP-TDC is illustrated in Fig. 11 [9]. The phase and frequency errors of the PLL are detected and digitalized by a PFD and an MP-TDC, respectively. The timing amplifier is applied to the MP-TDC to increase its timing resolution at low supply voltages. The circuit operation of the TA has been theoretically analysed [14]. When the timing difference $(\Delta T)$ of the PFD, between $U p$ and $D n$, is very small, it can be amplified to $G_{T A} \times \Delta T$ using a TDC with a TA $\left(G_{T A}\right)$. The MP-TDC uses multi-phase signals to sample the timing difference. The multi-phase signals are from the MP-DCO.

The timing diagram of the locking acquisition is also shown in Fig. 11. The locking acquisition mechanism of ADPLL can be theoretically considered as a sequence of three operations. The first is the fast-locking process that uses a coarse TDC (CTDC), as shown in Fig. 12(a). A CTDC can detect large phase differences. The CTDC is adopted to extend the operational range of an MP-TDC. Up and Dn signals are combined with an XOR gate to become the pulse signal Ps. Second, the timing resolution of the middle TDC (MTDC) is $1 / 8$ of the timing resolution of the CTDC. The phase difference of the MP-DCO defines the MTDC timing resolution. Fig. 12(b) presents the scheme of the MTDC. The target frequency can be achieved by the MTDC. Finally, the small phase difference can be digitized by the fine TDC (FTDC) with a TA as shown in Fig. 12(c). The FTDC with a TA expressed as:

$$
\Delta_{M P-T D C}=\left(\frac{T_{M P-D C O}}{8 \times G_{T A}}\right)
$$


where $T_{M P-D C O}$ is the period of the MP-DCO and $G_{T A}$ is the gain of TA using for the TDC. For these reasons, the proposed MP-TDC can be used to achieve a short locking time and superior frequency accuracy of ADPLL.

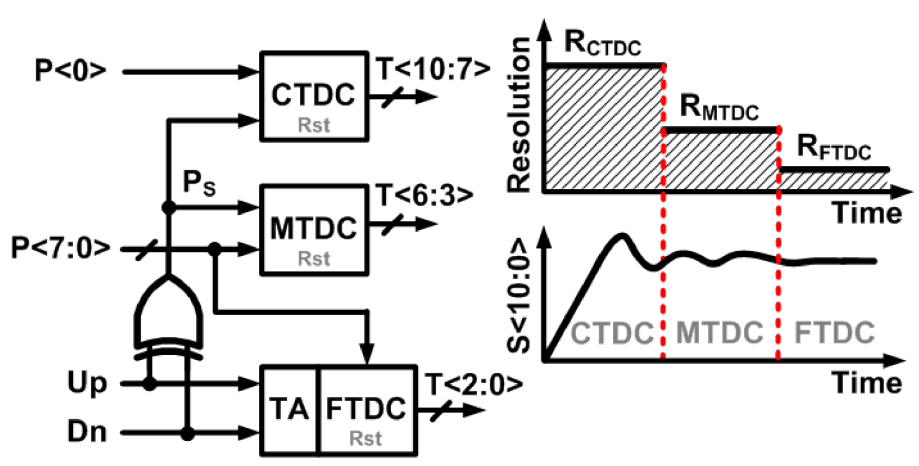

Fig. 11. Block and timing diagrams of proposed MP-TDC.
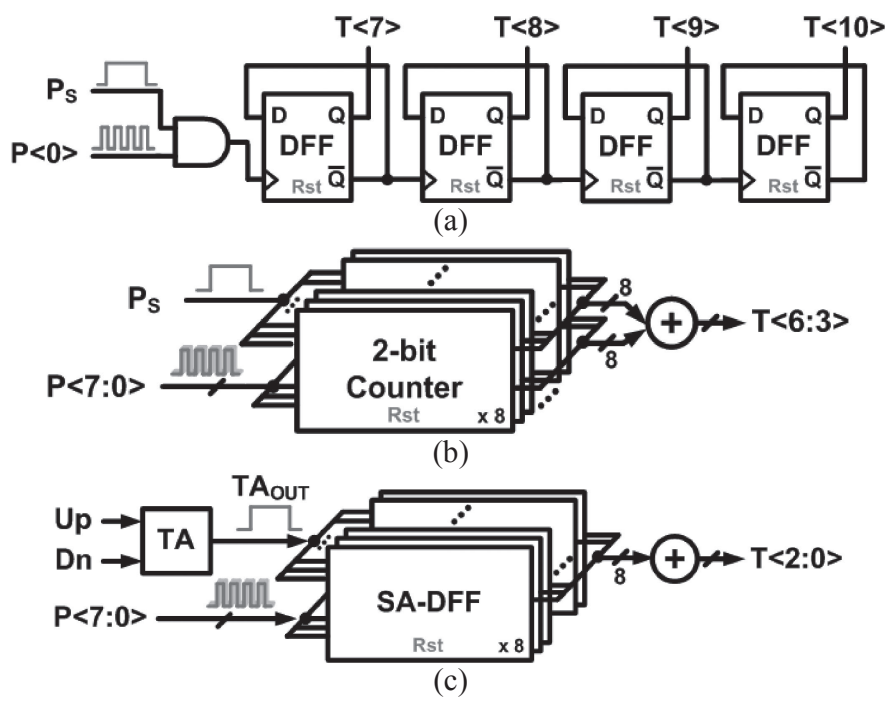

Fig. 12. (a) Coarse TDC (b) Middle TDC (c) Fine TDC.

The circuitry of TA is shown in Fig. 13 [2]. The TA consists of two DFFs, two SR latches, two buffers, three XOR gates and four MOS capacitances. The signals $U p$ and $D n$ feed the DFFs to produce two rising edges with very small time difference $(\Delta T)$. If the rising edges are applied to two inputs of SR latch at the same time, the latch becomes metastable. With the time amplifier, the time difference between can be enlarged to $G_{T A} \times \Delta T$. The gain of TA is expressed as:

$$
G_{T A}=\frac{2 C}{g_{m} T_{\text {OFF }}}
$$

where $C$ is the output capacitance of a NAND gate, $g_{m}$ is the transconductance of the NAND gate during the transitions and $T_{O F F}$ is the delay time of the buffer.

Fig. 14(a) shows the circuit diagram of the sense-amplifier latch (SA-latch). The bulk-controlled SA-latch can achieve a smaller sampling time than the conventional TSPC DFF circuits. The sum of the setup time and the hold time is $45 \mathrm{ps}$ and is smaller than that of TSPC-DFF (335 ps) under the Slow-Slow corner. 


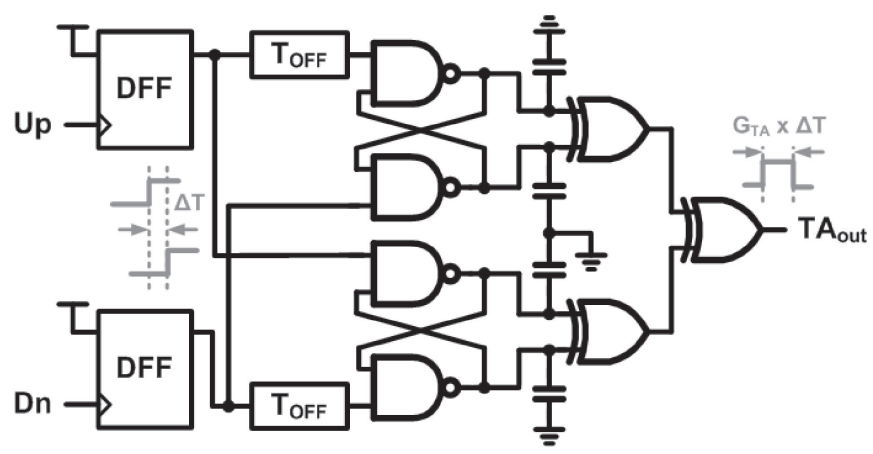

Fig. 13. Circuitry of TA. [2]

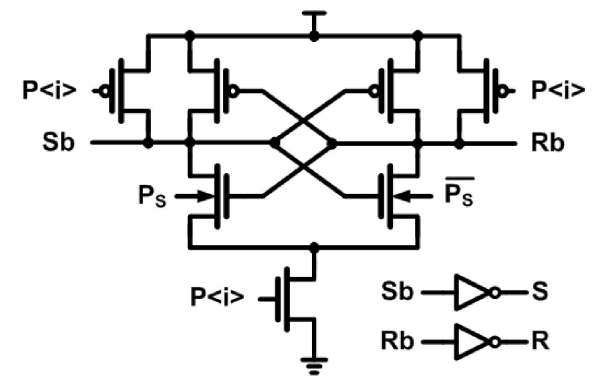

(a)

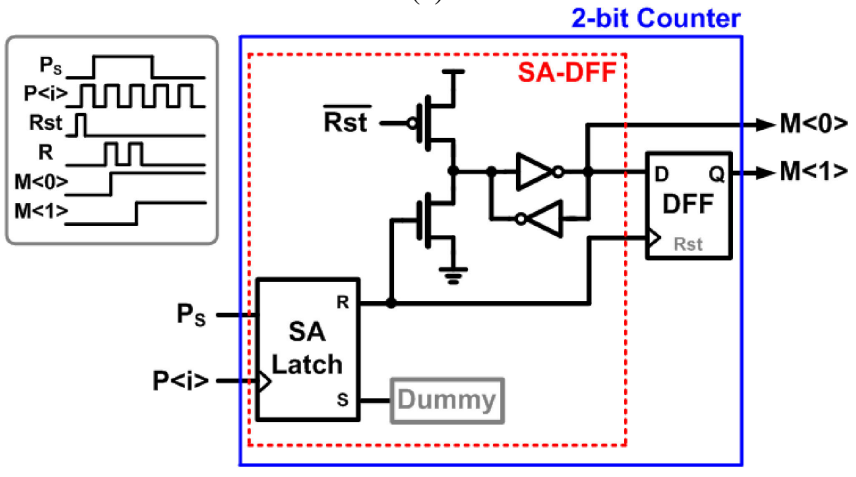

(b)

Fig. 14. (a) Circuit diagram of SA latch (b) 2-bit counter for MTDC.

The bulk-controlled technique is applied to compare the small input voltage difference. The 2-bit counter of the MTDC adopts the SA-DFF with a bulkcontrolled technique to sample the timing difference at a higher sampling clock rate. The SA-DFF with the bulk-controlled scheme is also used in the FTDC circuit to sample the phase difference. The bulk-controlled technique can be used to sample the timing difference at a low supply voltage. The SA-DFF with the bulkcontrolled scheme is also used in the FTDC circuit to sample the timing difference.

The measured timing range of the MP-TDC is designed by setting the bit number of the MP-TDC and the MP-DCO output frequency. When the TA gain is 5 and the bit number of the MP-TDC is 11 , the measured timing ranges are $10 \mathrm{~ns}$ and $17 \mathrm{~ns}$ when the DCO frequencies are $1.6 \mathrm{GHz}$ and $900 \mathrm{MHz}$, respectively. At $0.6 \mathrm{~V}$, the timing resolutions of MP-TDC are $27.8 \mathrm{ps}$ and $15.6 \mathrm{ps}$ at $900 \mathrm{MHz}$ and $1.6 \mathrm{GHz}$, respectively. 


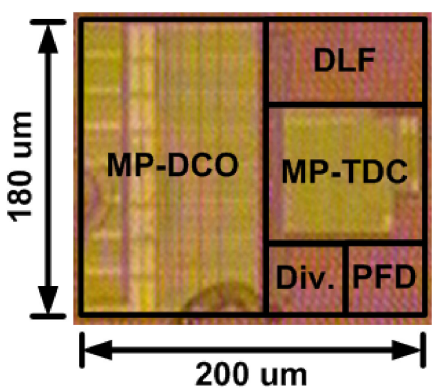

Fig. 15. Microphotograph of test chip.

\section{Experimental results}

The die photograph is displayed in the Fig. 15. Fig. 16 presents the jitter histograms at $0.6 \mathrm{~V}$. Its operational range is from $950 \mathrm{MHz}$ to $1.6 \mathrm{GHz}$ under a supply voltage of $0.6 \mathrm{~V}$. The RMS and peak-to-peak jitter (P2P jitter) at $950 \mathrm{MHz}$ and $1.6 \mathrm{GHz}$ are $3.7 \mathrm{ps}$ and $28.9 \mathrm{ps} ; 3.8 \mathrm{ps}$ and $33.7 \mathrm{ps}$, respectively. The power consumptions are $8.6 \mathrm{~mW}$ and $9.1 \mathrm{~mW}$ at $950 \mathrm{MHz}$ and $1.6 \mathrm{GHz}$, respectively. The jitter performances and power consumptions of ADPLL at $0.6 \mathrm{~V}$ is measured in Fig. 17. The I/O buffer and the coupling in the test chip can easily produce noise in the output signal, especially for the low voltage applications. The frequency errors are showed in Fig. 18. The mean frequency errors are less than $\pm 0.024 \%$. The worst frequency errors at $0.6 \mathrm{~V}$ are $\pm 3.1 \%$ under a varying output frequency. The RMS jitters are less than $0.71 \%$ below the varying output frequencies. For a fair comparison, the jitter has been normalized to the output period in Table II.

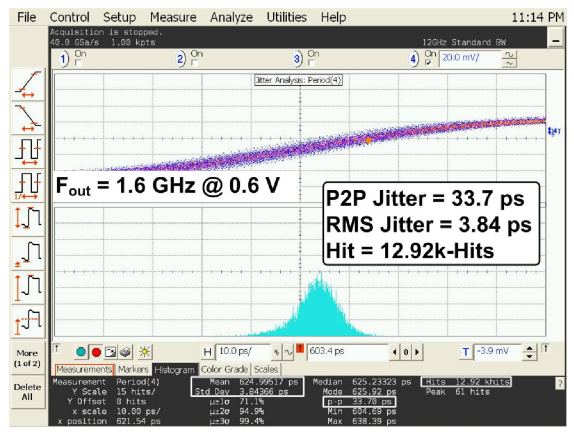

Fig. 16. Jitter performance at $1.6 \mathrm{GHz}$ 


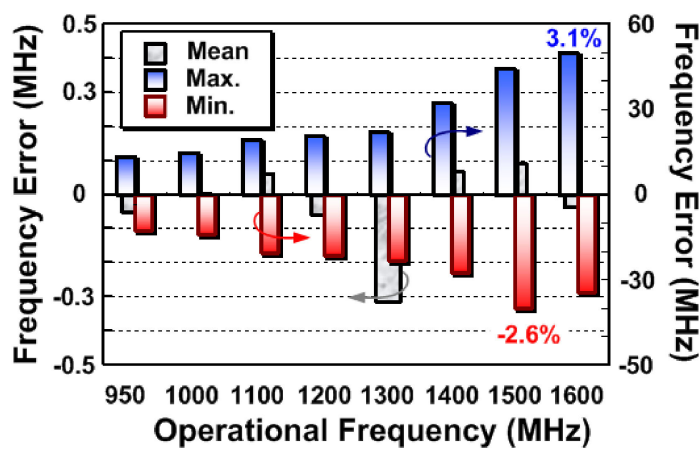

Fig. 18. Frequency errors of operational frequencies output at $0.6 \mathrm{~V}$.

\section{Conclusion}

This work presents an all-digital phase-locked loop (ADPLL) with 8-phase outputs under a low supply voltage. The ADPLL utilizes the proposed multi-phase digitally controlled oscillator (MP-DCO) and can reuse its 8-phase outputs in a time-todigital converter (TDC). This MP-DCO with two sub-feedback loops can achieve high operational frequencies. The multi-phase-based TDC with a time amplifier obtains a high timing resolution. Thus, this ADPLL has been implemented in a onepoly nine-metal (1P9M) $90 \mathrm{~nm}$ CMOS process and the operational frequency range is from $950 \mathrm{MHz}$ to $1.6 \mathrm{GHz}$ at $0.6 \mathrm{~V}$. This work achieves the highest operational frequency among prior published papers and offers multi-phase outputs in lower voltage operation as shown in Table II.

Table II. Performance Comparisons of ADPLL

\begin{tabular}{|c|c|c|c|c|c|}
\hline & {$[6]$} & {$[15]$} & {$[4]$} & {$[9]$} & This Work \\
\hline Technology (nm) & 130 & 90 & 65 & 65 & 90 \\
\hline Supply Voltage (V) & $0.6(0.6 \sim 1.6)$ & 0.6 & 0.5 & $1.1 / 1.3$ & 0.6 \\
\hline DCO Phase Number & 2 & 1 & 3 & 10 & 8 \\
\hline $\begin{array}{c}\text { Operating Frequency } \\
\text { (MHz) }\end{array}$ & $10 \sim 500$ & 800 & $90 \sim 1250$ & $600 \sim 800$ & $950 \sim 1600$ \\
\hline RMS Jitter (ps) & 39 & $\begin{array}{c}12.1 \\
@ 800 \mathrm{MHz}\end{array}$ & $\begin{array}{c}3 \\
@ 1 \mathrm{GHz}\end{array}$ & $\begin{array}{c}21.5 \\
@ 800 \mathrm{MHz}\end{array}$ & $\begin{array}{c}3.8 \\
@ 1.6 \mathrm{GHz}\end{array}$ \\
\hline P2P Jitter (ps) & $\begin{array}{c}288 \\
(191 \mathrm{MHz}\end{array}$ & $\begin{array}{c}80.5 \\
@ 800 \mathrm{MHz}\end{array}$ & - & $\begin{array}{c}193 \\
@ 800 \mathrm{MHz}\end{array}$ & $\begin{array}{c}33.7 \\
1.6 \mathrm{GHz}\end{array}$ \\
\hline Power (mW) & $7.2(1.2 \mathrm{~V})$ & $\begin{array}{c}0.66 \\
@ 800 \mathrm{MHz}\end{array}$ & $\begin{array}{c}1.65 \\
01 \mathrm{GHz}\end{array}$ & $\begin{array}{c}2.66 \mathrm{~mA} \\
@ 800 \mathrm{MHz}\end{array}$ & $\begin{array}{c}9.1 \\
@ 1.6 \mathrm{GHz}\end{array}$ \\
\hline Chip Area (mm $\left.{ }^{2}\right)$ & 0.09 & 0.02 & 0.03 & 0.027 & 0.036 \\
\hline RMS Jitter/Period (\%) & 0.74 & 0.97 & 0.3 & 1.72 & 0.61 \\
\hline
\end{tabular}

\section{Acknowledgments}

The authors would like to thank the Ministry of Science and Technology and the Chip Implementation Center (CIC), Taiwan (R.O.C) for fabricating the test chip. 